\title{
High Resolution Electronic Spectroscopy of $o$ - and $m$-Toluidine in the Gas Phase. Barrier Height Determinations for the Methyl Group Torsional Motions ${ }^{\dagger}$
}

\author{
Philip J. Morgan, Leonardo Alvarez-Valtierra, and David W. Pratt* \\ Department of Chemistry, University of Pittsburgh, Pittsburgh, Pennsylvania 15260
}

Received: April 30, 2009; Revised Manuscript Received: June 4, 2009

\begin{abstract}
High resolution electronic spectra of $o$ - and $m$-toluidine have each been recorded for the $\mathrm{S}_{1} \leftarrow \mathrm{S}_{0}$ origin band transitions of the isolated molecules. Each spectrum is split into two sub-bands owing to tunneling motions along the methyl group torsional coordinate. Analyses of these data provide information about the preferred configurations of the methyl groups and the barriers opposing their motions in both the ground and excited electronic states. Despite their apparent similarities, the experiments reveal that these properties are quite different in the two molecules. Possible reasons for this behavior are discussed.
\end{abstract}

\section{Introduction}

The subject of internal rotation has been studied extensively for many years, especially concerning molecules containing an attached methyl group. ${ }^{1-8}$ Of particular interest is the effect that different neighboring groups have on the potential barrier heights of a methyl group attached to a benzene ring. In toluene itself, internal rotation of the methyl group is relatively free in both the ground electronic state $\left(\mathrm{S}_{0}\right)$ and the lowest excited electronic state $\left(\mathrm{S}_{1}\right)$. The measured barriers are $\sim 5$ and $\sim 25 \mathrm{~cm}^{-1}$, respectively. ${ }^{6}$ However, substitution of a second ring hydrogen atom with a methoxy group changes this situation dramatically. A recent study of 2- and 3-methylanisole ${ }^{7}$ showed that the methyl group internal motions are significantly more rigid in one of the electronic states and less rigid in the other, with the corresponding barriers depending on the positions of the two groups relative to one another.

In this contribution, we report on a similar study of 2- and 3 -methylaniline ( $o$ - and $m$-toluidine) in which an amino group replaces the methoxy group in the methylanisoles. This problem has been addressed before by Ito and co-workers ${ }^{2}$ using low resolution fluorescence excitation spectroscopy (FES). These studies showed that the potential barrier of the methyl rotor in $o$-toluidine is very large in the ground state but very small in the first excited electronic state. The opposite behavior was observed for $m$-toluidine with nearly free internal rotation in the ground state and much more rigid internal rotation in the excited state. Okuyama et al. ${ }^{2}$ found no change in conformation of the methyl group upon excitation in either $o$ - or $m$-toluidine. It is the goal of the present work to determine if these conclusions are correct and to explore in greater detail the factors that might be responsible for these remarkable effects. Our tool is high resolution FES, as in the case of the methylanisoles. ${ }^{7}$

\section{Experimental Section}

Both $o$-toluidine and $m$-toluidine were purchased from Sigma Aldrich and used as received. Rotationally resolved $\mathrm{S}_{1} \leftarrow \mathrm{S}_{0}$ excitation spectra were recorded using the molecular beam laser spectrometer described elsewhere. ${ }^{9}$ Briefly, samples were heated

\footnotetext{
"Part of the "Robert W. Field Festschrift".

* To whom correspondence should be addressed. E-mail: pratt@ pitt.edu.

* Present address: División de Ciencias e Ingenierías, Universidad de Guanajuato, Campus León. León, Gto. 37150, México.
}

to $\sim 45{ }^{\circ} \mathrm{C}$ in a quartz source, seeded in dry argon gas $(>90 \%$ purity), and then expanded through a $240 \mu \mathrm{m}$ tip nozzle. The expansion was skimmed $\sim 2 \mathrm{~cm}$ downstream with a $1 \mathrm{~mm}$ diameter skimmer to form a molecular beam and then crossed $15 \mathrm{~cm}$ downstream of the nozzle with a laser beam operating in the UV. The laser radiation was generated by a ring dye laser operating in the visible (Rhodamine 590 dye) whose output was externally doubled using a Wavetrain frequency doubler (572 and $600 \mathrm{BBO}$ nm crystals) to produce $\sim 2 \mathrm{~mW}$ of UV radiation with a line width of $\sim 1 \mathrm{MHz}$. Fluorescence was collected with spatially selective optics and detected by a photon counting system and a PMT. All spectra were recorded using the jba95 data acquisition software. ${ }^{10}$ Typical scan lengths were $4 \mathrm{~cm}^{-1}$ over $1000 \mathrm{~s}$. The $\mathrm{I}_{2}$ absorption spectrum was used to determine the absolute transition frequencies of the excitation spectrum to an accuracy of $\pm 30 \mathrm{MHz}$. A stabilized etalon was used to produce relative frequency markers having a mode-matched free spectral range of $599.5040 \pm 0.0010 \mathrm{MHz}$ in the UV, corresponding to a free spectral range of $299.7520 \pm 0.0005 \mathrm{MHz}$ in the visible. The resulting spectra were fit using the jb95 leastsquares fitting program. ${ }^{11}$

Theoretical calculations were performed using the Gaussian 03 suite of programs ${ }^{12}$ to supplement the experiments. Geometry optimizations of both $o$-toluidine and $m$-toluidine in the ground state were performed at the MP2 level of theory with a 6-31G** basis set. Configuration interaction singles (CIS) calculations with a $6-31 \mathrm{G}^{* *}$ basis set were performed on the excited state.

\section{Results}

Figure 1 shows the vibrationally resolved fluorescence excitation spectra of $o$-toluidine and $m$-toluidine obtained by Ito and co-workers. ${ }^{5}$ In this figure, a labeling scheme of the vibrational bands $0 a_{1}, 1 e, 2 e$, and so forth is used where a number indexes the upper state torsional levels and a letter designates their symmetries. In the infinite barrier limit, the levels $0 a_{1}$ and $1 e, 2 e$ and $3 a_{1}$, and so forth are degenerate. Reduction of this barrier leads to a methyl group tunneling motion that lifts the degeneracy of the $0 a_{1}, 1 e ; 2 e, 3 a_{1}$; and so forth levels in both electronic states. The origin band of $o$-toluidine appears at $34316.9 \mathrm{~cm}^{-1}$, red shifted with respect to toluene by more than $3000 \mathrm{~cm}^{-1}$. The origin band of $m$-toluidine is red shifted by an even larger amount, appearing at $33820.2 \mathrm{~cm}^{-1}$. As assigned by Okuyama et al., ${ }^{4}$ the $1 \mathrm{e}$ band 

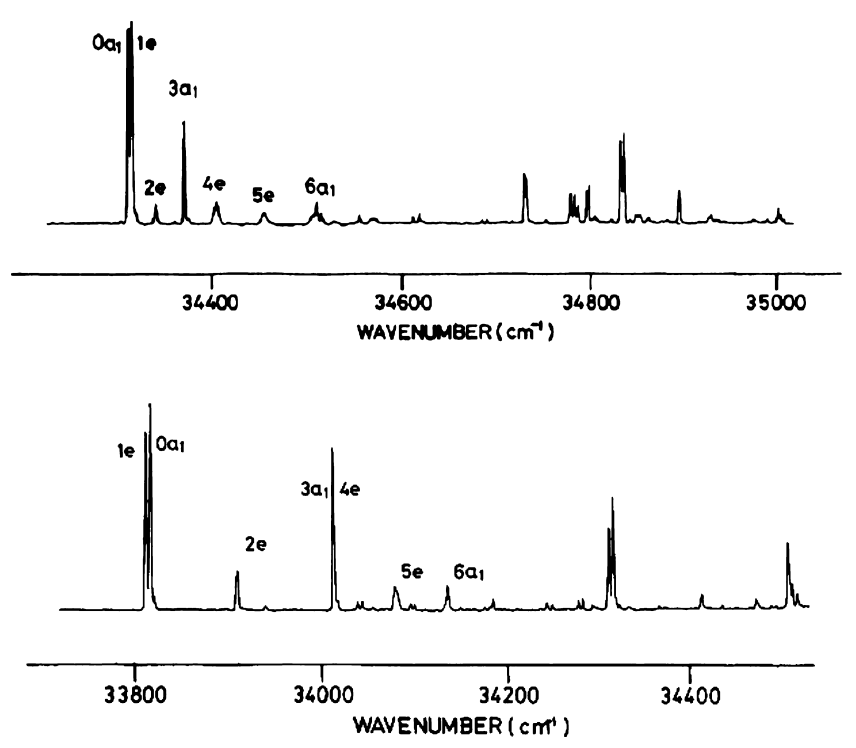

Figure 1. Vibrationally resolved fluorescence excitation spectra of $o$-toluidine (top) and $m$-toluidine (bottom) (ref 2).

is blue shifted with respect to the $0 \mathrm{a}_{1}$ band for $o$-toluidine but is red-shifted with respect to the $0 \mathrm{a}_{1}$ band for $m$-toluidine. These assignments suggest that the potential barriers for the methyl group torsional motion in both electronic states are significantly influenced by the relative positions of the methyl and amino groups on the benzene ring.

High resolution experiments have been performed to validate these conclusions. Figure 2 shows the rotationally resolved $\mathrm{S}_{1} \leftarrow \mathrm{S}_{0}$ fluorescence excitation spectrum of band $0 \mathrm{a}_{1}$ in $o$ toluidine. The spectrum spans approximately $2.9 \mathrm{~cm}^{-1}$ and exhibits both $a$ - and $b$-type transitions. The spectrum was fit using rigid-rotor Hamiltonians ${ }^{13}$ for both electronic states, confirming its identity as the $0 \mathrm{a}_{1}$ vibronic band. First, a simulated spectrum was generated using estimated rotational constants from the ab initio calculations. Single transitions from the simulated spectrum were then assigned to corresponding transi-

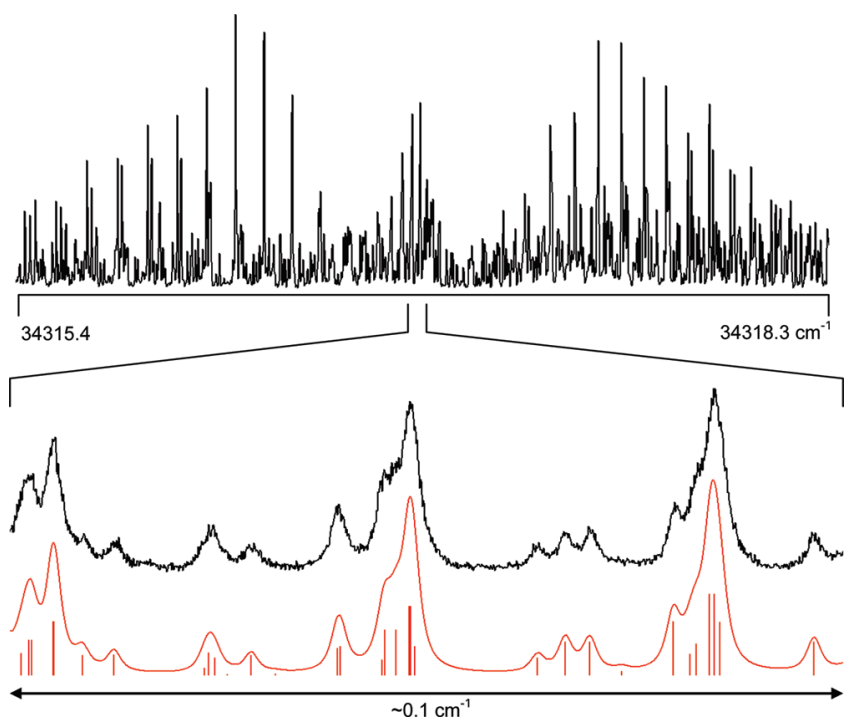

Figure 2. Rotationally resolved fluorescence excitation spectrum of the $0 \mathrm{a}_{1}$ band of $o$-toluidine with origin at $34316.9 \mathrm{~cm}^{-1}$. The lower part of the figure shows an expanded view of the Q-branch region. In this view, the upper trace is the experimental spectrum, and the lower trace is the simulated spectrum. Individual lines represent the transitions responsible for the spectrum. The band was fit using a Voigt line shape profile.
TABLE 1: Rotational Constants of $o$-Toluidine in Its Ground and Excited Electronic States ${ }^{a}$

\begin{tabular}{lccc}
\hline \multicolumn{1}{c}{ parameter } & $o$-toluidine $0 \mathrm{a}_{1}$ & $o$-toluidine $1 \mathrm{e}^{b}$ & theoretical $^{c}$ \\
\hline$A^{\prime \prime}(\mathrm{MHz})$ & $3230.9(1)$ & $3228.5(1)$ & 3243.6 \\
$B^{\prime \prime}(\mathrm{MHz})$ & $2189.0(1)$ & $2189.1(1)$ & 2185.9 \\
$C^{\prime \prime}(\mathrm{MHz})$ & $1316.8(1)$ & $1316.9(1)$ & 1318.7 \\
$\Delta A(\mathrm{MHz})$ & $-74.3(1)$ & $-113.4(1)$ & -91.5 \\
$\Delta B(\mathrm{MHz})$ & $-1.8(1)$ & $-8.3(1)$ & 21.1 \\
$\Delta C(\mathrm{MHz})$ & $-20.8(1)$ & $-20.5(1)$ & -9.8 \\
$\Delta I^{\prime \prime}\left(\mathrm{amu} \AA^{2}\right)$ & -3.51 & -3.65 & -3.77 \\
$\Delta I^{\prime}\left(\mathrm{amu} \AA^{2}\right)$ & -1.21 & -4.15 & -3.21 \\
$\mathrm{TM}$ angle to & $\pm 40(2)^{\circ}$ & $\pm 40(2)^{\circ}$ & $+42^{\circ}$ \\
$a$-inertial axis & & &
\end{tabular}

${ }^{a}$ Numbers in parentheses are the standard deviations of the last significant figure. ${ }^{b}$ See Table 3 for additional information about the first-order torsion-rotation perturbation coefficients that were used in the fit of this spectrum. ${ }^{c}$ Geometry optimization calculations done at the MP2/6-31G** and CIS/6-31G** levels of theory.

tions in the experimental spectrum, using the fitting program jb95. ${ }^{11} \mathrm{~A}$ linear least-squares fitting procedure was finally used to optimize the rotational constants from a comparison of the observed and calculated transitions. The bottom of Figure 2 shows a portion of the fit spectrum, where a standard deviation of $1.40 \mathrm{MHz}$ was obtained by fitting 135 lines. The band was found to be a hybrid band, having 59\% a-type and $41 \%$-type transition character. The spectrum was fit using a Voigt line shape profile for individual transitions with Gaussian widths of $20 \mathrm{MHz}$ and Lorentzian widths of $38 \mathrm{MHz}$, corresponding to a fluorescence lifetime of $\tau=4.2 \mathrm{~ns}$. Table 1 lists all of the inertial parameters obtained from the fit of the spectrum.

Band 1e of $o$-toluidine located at $34320.2 \mathrm{~cm}^{-1}$ is shown in Figure 3 and spans approximately $2.8 \mathrm{~cm}^{-1}$. Unlike the $0 \mathrm{a}_{1}$ band in this molecule, the high resolution spectrum of the 1e band in $o$-toluidine exhibits further splittings of the individual rovibronic transitions into two components. This confirms its identity as the 1e band. The normally degenerate rovibronic transitions in this band are coupled by the torsion-rotation interaction, leading to a K-dependent splitting in the high resolution spectrum. ${ }^{14}$ These splittings are described by the

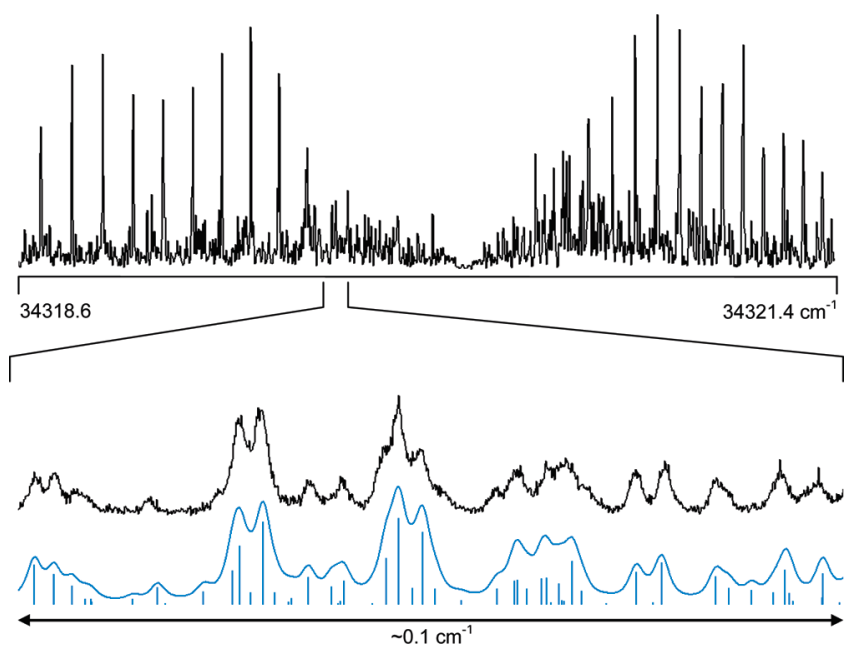

Figure 3. Rotationally resolved fluorescence excitation spectrum of the $1 \mathrm{e}$ band of $o$-toluidine with origin at $34320.2 \mathrm{~cm}^{-1}$. The lower part of the figure shows an expanded view of the P-branch region. In this view, the upper trace is the experimental spectrum, and the lower trace is the simulated spectrum. Individual lines represent the transitions responsible for the spectrum. The band was fit using a Voigt line shape profile. 


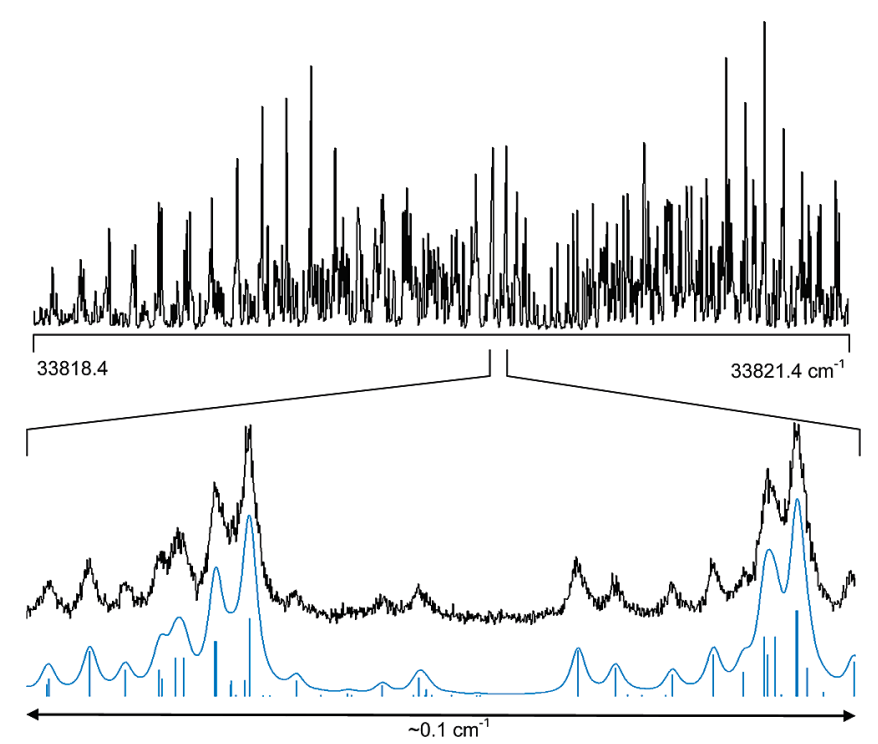

Figure 4. Rotationally resolved fluorescence excitation spectrum of the $0 \mathrm{a}_{1}$ band of $m$-toluidine with origin at $33820.2 \mathrm{~cm}^{-1}$. The lower part of the figure shows an expanded view of the Q-branch region. In this view, the upper trace is the experimental spectrum, and the lower trace is the simulated spectrum. Individual lines represent the transitions responsible for the spectrum. The band was fit using a Voigt line shape profile.

following "non-rigid" rotor Hamiltonian for both electronic states: ${ }^{3,13}$

$$
H_{\mathrm{eff}}^{\mathrm{E}}=A_{\mathrm{E}} P_{a}^{2}+B_{\mathrm{E}} P_{b}^{2}+C P_{c}^{2}+F W_{\mathrm{E}}^{(1)}\left(\rho_{a} P_{a}+\rho_{b} P_{b}\right)
$$

Here, $A_{\mathrm{E}}$ and $B_{\mathrm{E}}$ are the effective rotational constants given by

$$
A_{\mathrm{E}}=A+F W_{\mathrm{E}}^{(2)} \rho_{a}^{2}, \quad B_{\mathrm{E}}=B+F W_{\mathrm{E}}^{(2)} \rho_{b}^{2}
$$

$F$ is the internal rotor constant, $W_{\mathrm{E}}^{(1)}$ and $W_{\mathrm{E}}^{(2)}$ are the first- and second-order perturbation terms, first written down by Herschbach, ${ }^{15}$ and $\rho_{a}$ and $\rho_{b}$ are weighted direction cosines of the angle between the axis of internal rotation and the $a$ - and $b$-inertial axes of the molecule, where $\rho_{a}=\lambda_{a}\left(I_{\alpha} / I_{a}\right)$. $I_{\alpha}$ is the moment of inertia of the methyl group, and $r$ is a reduction factor

$$
r=1-\sum\left(\lambda_{g}^{2} I_{\alpha} / I_{g}\right), \quad g=a, b, c
$$

A portion of the fit spectrum of the 1e band of $o$-toluidine is shown at the bottom of Figure 3; a standard deviation of 7.82 $\mathrm{MHz}$ was obtained from a fit of 100 lines. The inertial parameters obtained from the fit of the spectrum are also listed in Table 1. The inertial parameters obtained for the 1e band of $o$-toluidine are consistent with those obtained by Kalkman and Meerts. ${ }^{16}$

The rotationally resolved spectrum of the $0 \mathrm{a}_{1}$ band of $m$-toluidine spans approximately $3.0 \mathrm{~cm}^{-1}$ and is shown in Figure 4. No additional splittings appear in this spectrum. Hence, it was fit using rigid-rotor Hamiltonians for both electronic states, confirming its identity as an $\mathrm{a}_{1}$ vibronic band. The bottom of Figure 4 shows a portion of the fit at full experimental resolution; 153 lines were assigned and a standard deviation of $1.73 \mathrm{MHz}$ was obtained. The spectrum was again fit with a Voigt line shape profile with Gaussian widths of $20 \mathrm{MHz}$ and
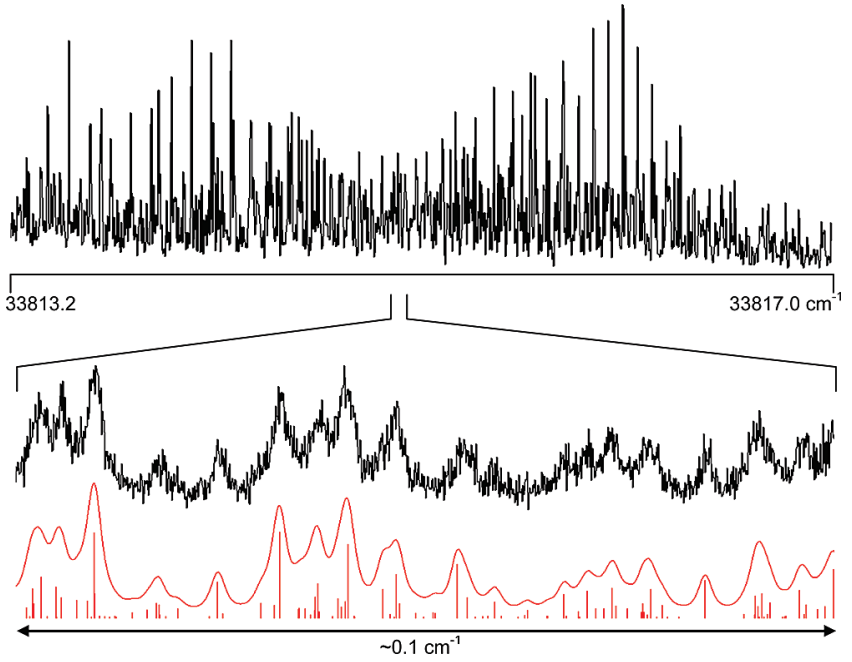

Figure 5. Rotationally resolved fluorescence excitation spectrum of the 1e band of $m$-toluidine with origin at $33814.9 \mathrm{~cm}^{-1}$. The lower part of the figure shows an expanded view of the Q-branch region. In this view, the upper trace is the experimental spectrum, and the lower trace is the simulated spectrum. Individual lines represent the transitions responsible for the spectrum. The band was fit using a Voigt line shape profile.

Lorentzian widths of $38 \mathrm{MHz}$, corresponding to a lifetime of $\tau$ $=4.2 \mathrm{~ns}$. The origin band of $m$-toluidine was also found to be as hybrid band with $48 \%$ a-type and $52 \%$ b-type transition character. The inertial parameters obtained from the fit of the spectrum are listed in Table 2.

Figure 5 shows the rotationally resolved spectrum of the $1 \mathrm{e}$ band of $m$-toluidine located at $33814.9 \mathrm{~cm}^{-1}$. As in the case of the 1 e band for $o$-toluidine, this band could not be fit using a rigid-rotor Hamiltonian and required the addition of perturbation terms. This is again a result of the torsion-rotation interaction lifting the degeneracies of certain rovibronic transitions, confirming the identity of this vibronic band as the 1e band. The Hamiltonian in eq 1 was again used to obtain a fit with a standard deviation of $3.24 \mathrm{MHz}$ for the 115 assigned lines. A comparison of the simulated spectrum to the experimental shows excellent agreement and can be seen in the bottom of Figure 5. The inertial parameters obtained from the fit are also listed in Table 2. Neither 1e band required the use of Watson distortion terms in the fit. ${ }^{17}$

\section{Discussion}

4.1. Ground and Excited State Conformers. Tables 1 and 2 list the ground and excited state rotational constants for both

TABLE 2: Rotational Constants of $m$-Toluidine in Its Ground and Excited Electronic States ${ }^{a}$

\begin{tabular}{lccc}
\hline \multicolumn{1}{c}{ parameter } & $m$-toluidine $0 \mathrm{a}_{1}$ & $m$-toluidine $1 \mathrm{e}^{b}$ & theoretical $^{c}$ \\
\hline$A^{\prime \prime}(\mathrm{MHz})$ & $3701.3(1)$ & $3700.2(1)$ & 3637.7 \\
$B^{\prime \prime}(\mathrm{MHz})$ & $1795.9(1)$ & $1795.4(1)$ & 1796.3 \\
$C^{\prime \prime}(\mathrm{MHz})$ & $1210.4(1)$ & $1210.3(1)$ & 1211.5 \\
$\Delta A(\mathrm{MHz})$ & $-164.7(1)$ & $-164.9(1)$ & -46.5 \\
$\Delta B(\mathrm{MHz})$ & $-7.1(1)$ & $-7.0(1)$ & 9.0 \\
$\Delta C(\mathrm{MHz})$ & $-12.9(1)$ & $-12.9(1)$ & -1.3 \\
$\Delta I^{\prime \prime}\left(\mathrm{amu} \AA^{2}\right)$ & -0.414 & -0.508 & -3.12 \\
$\Delta I^{\prime}\left(\right.$ amu $\left.\AA^{2}\right)$ & -3.40 & -3.50 & -3.06 \\
$\mathrm{TM}$ angle to & $\pm 46(2)^{\circ}$ & $\pm 46(2)^{\circ}$ & $+45^{\circ}$
\end{tabular}

$a$-inertial axis

${ }^{a}$ Numbers in parentheses are the standard deviations of the last significant figure. ${ }^{b}$ See Table 3 for additional information about the first-order torsion-rotation perturbation coefficients that were used in the fit of this spectrum. ${ }^{c}$ Geometry optimization calculation done at the MP2/6-31G** and CIS/6-31G** levels of theory. 

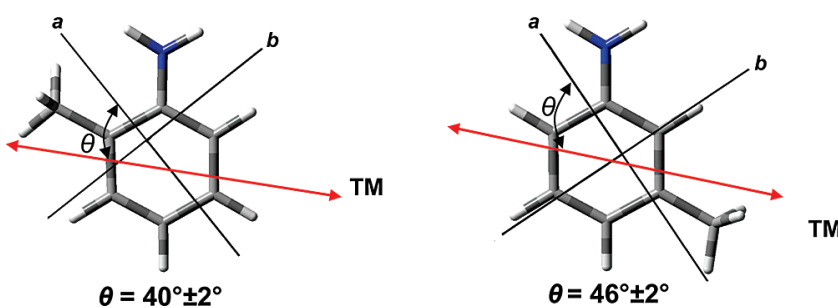

Figure 6. Ground-state structures and $\mathrm{S}_{1} \leftarrow \mathrm{S}_{0} \mathrm{TM}$ orientation vectors in the $o$ - and $m$-toluidines.

origin bands of $o$-toluidine and $m$-toluidine, respectively, along with the corresponding theoretical values. Comparisons between the theoretical and experimental rotational constants of both molecules confirm their respective identities. It can be seen that the set of rotational constants obtained for both $o$ - and $m$-toluidine are very different from one another.

These differences in the values of the ground and excited rotational constants can be easily understood from Figure 6, which depicts the ground state molecular structures of $o$ - and $m$-toluidine in their $a b$ inertial planes. The $a$ and $b$ axes are rotated with respect to their "canonical" positions owing to the simultaneous presence of the $-\mathrm{NH}_{2}$ and $-\mathrm{CH}_{3}$ groups. In $o$-toluidine, the $a$ axis lies between the two substituents, passing through the connecting ring $\mathrm{C}-\mathrm{C}$ bond, whereas in $m$-toluidine, the $b$ axis lies between them, passing through the adjacent $\mathrm{C}-\mathrm{H}$ bond. As a result, the $-\mathrm{NH}_{2}$ group in $m$-toluidine lies closer to the $a$ inertial axis, giving it the larger $A$ value. Correspondingly, the $-\mathrm{NH}_{2}$ group in $o$-toluidine lies closer to the $b$ axis, giving it the larger $B$ value. The $C$ value of $o$-toluidine is larger than that of $m$-toluidine owing to the displacement of its COM relative to the center of the ring.

The ground state inertial defect values of the $0 \mathrm{a}_{1}$ and $1 \mathrm{e}$ methyl torsional bands in $o$-toluidine are close to the value expected for an attached methyl group $\left(-3.30 \mathrm{amu} \AA^{2}\right),-3.51$ and -3.65 amu $\AA^{2}$, respectively. (The slightly more negative values of $\Delta \mathrm{I}$ can be attributed to some nonplanar character of the attached $-\mathrm{NH}_{2}$ group.) However, the inertial defect values of the $0 \mathrm{a}_{1}$ and $1 \mathrm{e}$ methyl torsional bands in $m$-toluidine are significantly less in magnitude, -0.414 and $-0.508 \mathrm{amu} \AA^{2}$, respectively. Moreover, the $\Delta \mathrm{I}$ value of the $0 \mathrm{a}_{1}$ level in $o$-toluidine decreases in magnitude to $-1.21 \mathrm{amu} \AA^{2}$, the $\Delta \mathrm{I}$ value of the 1e level in $o$-toluidine increases in magnitude to $-4.15 \mathrm{amu} \AA^{2}$, and the $\Delta \mathrm{I}$ values of the $0 \mathrm{a}_{1}$ and $1 \mathrm{e}$ level in $m$-toluidine increase in magnitude to -3.40 and -3.50 amu $\AA^{2}$, respectively, when the molecules are excited to their $S_{1}$ states.

Figure 6 also shows the $S_{1} \leftarrow S_{0}$ transition moment (TM) orientations in the inertial frames of both molecules. Two orientations are possible, making angles of approximately $\theta=$ $\pm 45^{\circ}$ with respect to $a$ in both $o$ - and $m$-toluidine. Taking $\theta$ to be negative orients the TM vector approximately parallel to the $\mathrm{C}-\mathrm{N}$ bond in both molecules, whereas taking $\theta$ to be positive orients the TM vector approximately perpendicular to the $\mathrm{C}-\mathrm{N}$ bond in both molecules. If the $\mathrm{S}_{1}$ states of $o$ - and $m$-toluidine are both $L_{\mathrm{b}}$ states, then the perpendicular orientation is expected if the $-\mathrm{NH}_{2}$ group is the "dominant" substituent.

The molecular orbitals (MOs) that participate in the $\mathrm{S}_{1} \leftarrow \mathrm{S}_{0}$ transitions of the two molecules are shown in Figure 7, together with the corresponding MOs of toluene and aniline. Each transition is predicted by theory to be primarily highest occupied molecular orbital (HOMO)-lowest unoccupied molecular orbital (LUMO) in nature. But the relevant MOs in $o$ - and $m$-toluidine are clearly much more similar to those of aniline than to those of toluene. The TM orientations are also similar,

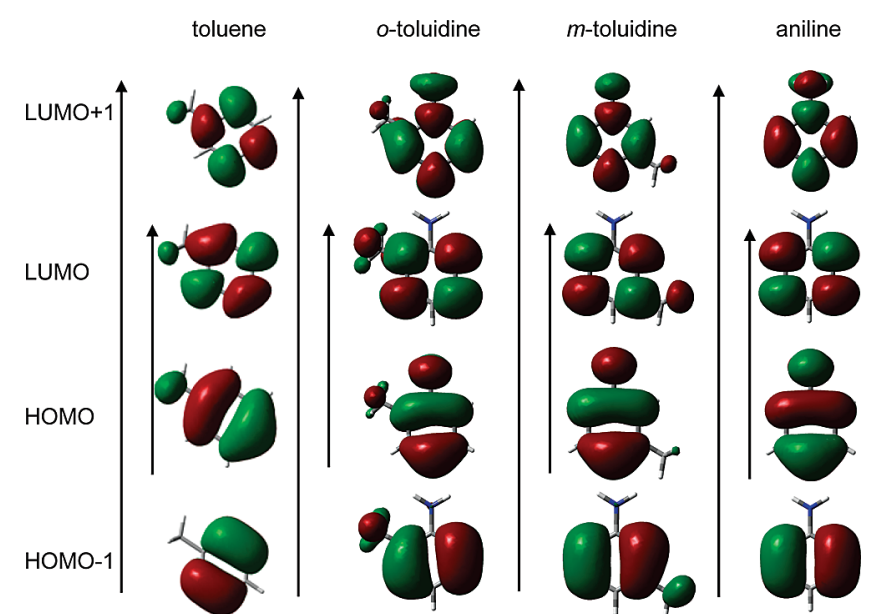

Figure 7. Frontier molecular orbitals calculated at the CIS/6-31G(d,p) level of toluene, $o$-toluidine, $m$-toluidine, and aniline. All transitions are primarily $\mathrm{HOMO} \rightarrow$ LUMO.

as is clear from Figure 7. Thus, we conclude that the amino group is the dominant substituent in the two molecules, and that the $\mathrm{S}_{1} \leftarrow \mathrm{S}_{0} \mathrm{TM}$ vector is oriented approximately perpendicular to the $\mathrm{C}-\mathrm{N}$ bond in both species.

Experimental confirmation of this conclusion can be done using an analysis first carried out by Plusquellic and Pratt. ${ }^{18}$ If we take the inertial axis frames to be those shown in Figure 6, we know that the torsion-rotation parameters $D_{a}$ and $D_{b}$, and $\rho_{a}$ and $\rho_{b}$ must have the same signs in this configuration (cf. Table 3). Therefore, either $\rho_{a}>0$ and $\rho_{b}>0$ or $\rho_{a}<0$ and $\rho_{b}$ $<0$, depending on the sense of rotation of the methyl rotor relative to its own $C_{3}$ axis. It has been found in the previous study that the line positions in the computed $\mathrm{E} \leftrightarrow \mathrm{E}$ torsional bands are insensitive to the choice of signs, but that the calculated line intensities are sensitive to choice of signs. Therefore, the correct relative intensities of the $a$ - and $b$-type lines would be given by either $\rho_{a}>0$ and $\rho_{b}>0$ or $\rho_{a}<0$ and $\rho_{b}<0$ if $\theta_{\mathrm{TM}}>0$, and $\rho_{a}<0$ and $\rho_{b}>0$ or $\rho_{a}>0$ and $\rho_{b}<0$ if $\theta_{\mathrm{TM}}<0$. Since we know that $\rho_{a}$ and $\rho_{b}$ must have the same signs, we can deduce that the $\theta_{\mathrm{TM}}$ must be positive, which is also the sign choice that gives the correct relative intensities. Theory agrees with these determinations (Tables 1 and 2).

Upon excitation, both molecules exhibit a large decrease in the $A$ rotational constant and much smaller decreases in $B$ (and $C)$; $\Delta A \sim-100$ and $\Delta B \sim-10 \mathrm{MHz}$ in $o$-toluidine and $\Delta A \sim$ -150 and $\Delta B \sim-10 \mathrm{MHz}$ in $m$-toluidine. Aniline, by contrast,

TABLE 3: First-Order Torsion-Rotation Perturbation Coefficients in the Hamiltonian and Deduced Barrier Heights for $e$-Symmetry Torsional Bands in $o$ - and $m$-Toluidine ${ }^{a}$

\begin{tabular}{lcc}
\hline \multicolumn{1}{c}{ parameter } & $o$-toluidine 1e band & $m$-toluidine 1e band \\
\hline$D_{a}^{\prime \prime}(\mathrm{MHz})$ & $0.23^{b}$ & $6406.1(1)$ \\
$D_{b}^{\prime \prime}(\mathrm{MHz})$ & $0.069^{b}$ & $1719.7(1)$ \\
$W^{(1)^{\prime \prime}}$ & $7.64 \times 10^{-5}$ & 1.911 \\
$s^{\prime \prime}$ & 59.202 & 0.769 \\
$V_{3}^{\prime \prime}\left(\mathrm{cm}^{-1}\right)$ & $703^{b}$ & 9.45 \\
$D_{a}^{\prime}(\mathrm{MHz})$ & $2628.4(1)$ & $21.5(1)$ \\
$D_{b}{ }^{\prime}(\mathrm{MHz})$ & $1004.7(1)$ & $26.4(30)$ \\
$W^{(1) \prime}$ & 0.873 & 0.006 \\
$s^{\prime}$ & 3.702 & 27.269 \\
$V_{3}^{\prime}\left(\mathrm{cm}^{-1}\right)$ & 44.9 & 322
\end{tabular}

${ }^{a}$ Numbers in parentheses are the standard deviations of the last significant figure. ${ }^{b}$ Torsion-rotation terms derived from an assumed barrier height of $703 \mathrm{~cm}^{-1}$ (ref 2). 

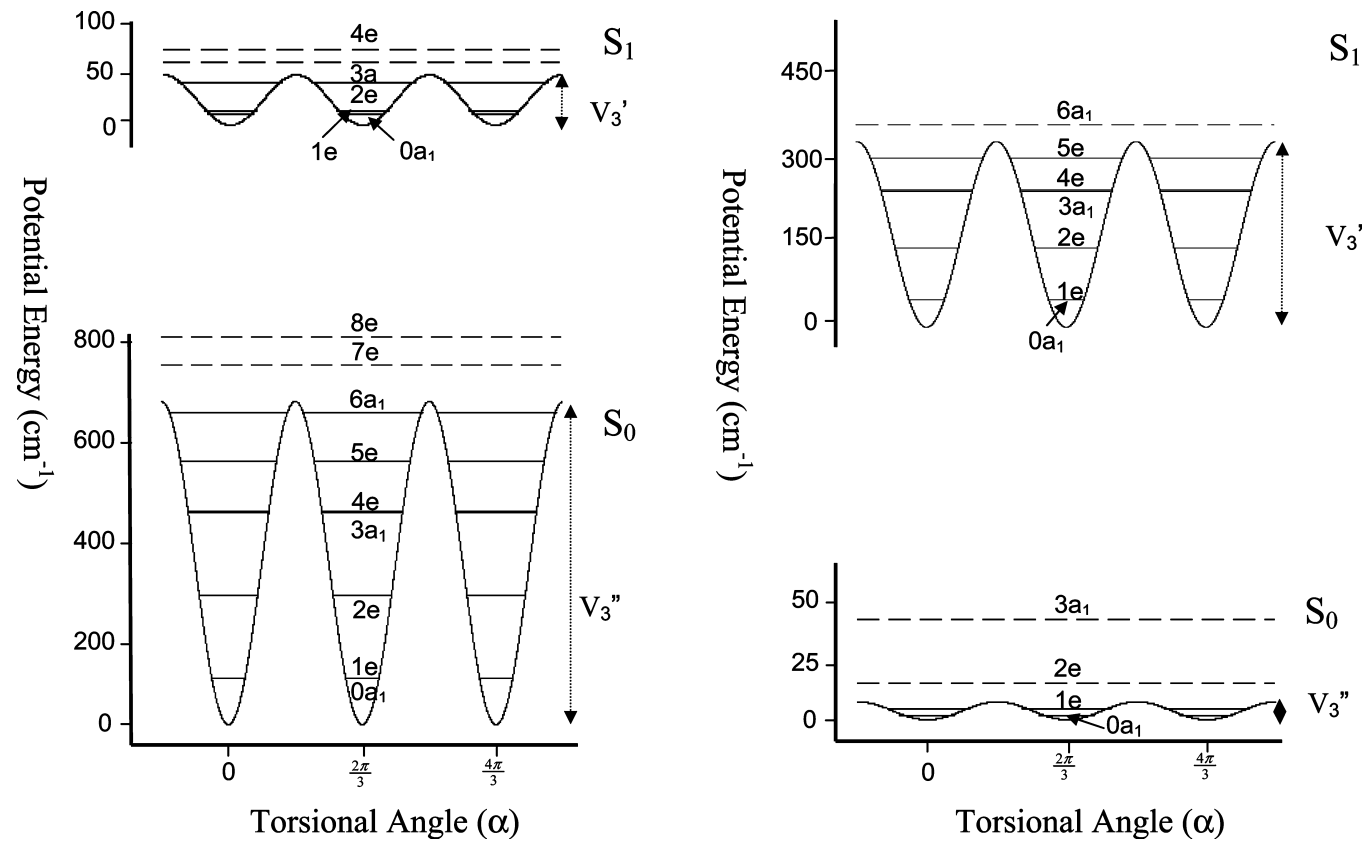

Figure 8. Torsional energy curves of $o$-toluidine (left) and $m$-toluidine (right).

has $\Delta A \sim-300$ and $\Delta B \sim+40 \mathrm{MHz} .{ }^{19}$ The changes in aniline have their origin in a quinoidal distortion of the $S_{1}$ state; the "perpendicular" $\mathrm{C}-\mathrm{C}$ ring bonds are longer than the "parallel" ones. The smaller (in-magnitude) values $\Delta A$ and $\Delta B$ in the toluidines, in which similar distortions are occurring, may be attributed to the tilts of their inertial axes with respect to those of aniline. Curiously, the values of $\Delta A$ and $\Delta B$ are significantly different in the $0 \mathrm{a}_{1}$ and 1e torsional levels of the $S_{1}$ state of $o$-toluidine.

4.2. Methyl Torsional Barriers. The torsional parameters $D_{g}\left(=F W_{\mathrm{E}}^{(1)} \rho_{g}, g=a, b, c\right)$ obtained from the fits of the $1 \mathrm{e}$ bands of $o$ - and $m$-toluidine are listed in Table 3. Using Herschbach's tables, both the reduced barrier heights $(s)$ and the corresponding 3-fold barriers $V_{3}(=(9 / 4) F \cdot s)$ could be calculated. Because of the very large barrier in the ground electronic state, values of the torsion-rotation terms for $o$ toluidine in the $\mathrm{S}_{0}$ state were not experimentally measured. Instead, they were calculated from the barrier height of $V_{3}^{\prime \prime}=$ $703 \mathrm{~cm}^{-1}$ obtained by Okuyama et al. ${ }^{2}$ (Barrier heights much larger than $500 \mathrm{~cm}^{-1}$ do not produce measurable perturbations at our experimental resolution.) A reduced barrier height of 59.202 was calculated and the first-order perturbation coefficient $\left(W_{\mathrm{E}}^{(1)}=7.64 \times 10^{-5}\right)$ was obtained through interpolation, yielding torsion-rotation terms of $D_{a}=0.23$ and $D_{b}=0.069$ $\mathrm{MHz}$. In the $\mathrm{S}_{1}$ state, the torsion-rotation terms were found to be much larger and could be determined by experiment $\left(D_{a}=\right.$ 2628.4 and $D_{b}=1004.7 \mathrm{MHz}$ ). The reduced barrier height was obtained through interpolation of the first-order perturbation coefficient $\left(W_{\mathrm{E}}^{(1)}=0.873\right)$ and a value of $3.702\left(V_{3}^{\prime}=44.89\right.$ $\mathrm{cm}^{-1}$ ) was calculated.

Interestingly, the opposite behavior was observed in $m$ toluidine, where the torsion-rotation terms in the $\mathrm{S}_{0}$ state are quite large $\left(D_{a}=6406.1\right.$ and $\left.D_{b}=1719.7 \mathrm{MHz}\right)$ but much smaller in the $\mathrm{S}_{1}$ state $\left(D_{a}=21.5\right.$ and $\left.D_{b}=26.4 \mathrm{MHz}\right)$. This resulted in calculated reduced barrier heights of $0.769\left(V_{3}=\right.$ $\left.9.45 \mathrm{~cm}^{-1}\right)$ in the ground state and $27.269\left(V_{3}^{\prime}=322.23 \mathrm{~cm}^{-1}\right)$ in the excited state. These results are consistent with the finding that the $1 \mathrm{e}$ band is blue shifted with respect to the $0 \mathrm{a}_{1}$ origin band in $o$-toluidine and red shifted in $m$-toluidine. The corresponding potential energy curves for both $o$ - and $m$-toluidine are shown in Figure 8.
As mentioned earlier, the observed inertial defect values of $o$-toluidine in the excited state $\left(\sim-1.2 \mathrm{amu} \AA^{2}\right)$ and $m$-toluidine in the ground state $\left(\sim-0.5\right.$ amu $\left.\AA^{2}\right)$ are much smaller than the expected value for a methyl group attached to an aromatic plane $\left(-3.3 \mathrm{amu} \AA^{2}\right)$. It can be seen that the small inertial defect values occur whenever the 3 -fold potential barrier heights are small, while the expected and larger values occur when the barrier heights are much larger. This general trend has been observed before, most notably in 2- and 3-methylanisole. ${ }^{3}$ Because of the planarity of the ring to which the methyl group is attached, the inertial defect is zero in the free rotor limit for an internal rotor that is collinear with an inertial axis of the molecule. This also holds to a good approximation for an arbitrary orientation of the symmetry axis of the methyl group in the symmetry plane of the molecule. ${ }^{20}$ The smaller the barrier height, the more the inertial defect value approaches zero, as observed in both molecules.

The barrier heights obtained for the ground and excited states of both molecules are consistent with those measured by Okuyama et al. ${ }^{2}$

4.3. Origins of the Torsional Barriers. As has been previously shown in the methylpyrimidines ${ }^{2}$ and the methylanisoles, ${ }^{3}$ the preferred configuration (staggered or eclipsed) and the potential energy curve along the torsional coordinate have their origins in two factors, the position of the methyl group relative to other ring substituents and differences in the $\pi$-bond orders of the adjacent $\mathrm{C}-\mathrm{C}$ bonds. (The staggered and eclipsed configurations are shown below in Scheme 1, where $\mathrm{S}$ denotes the attached substituent in the plane of the benzene ring.) According to theory, the methyl group in $o$-toluidine is staggered, whereas the methyl group in $m$-toluidine is eclipsed, in the electronic ground states of the two molecules. The

\section{SCHEME 1}

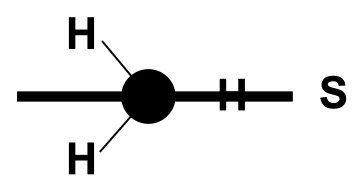

Eclipsed

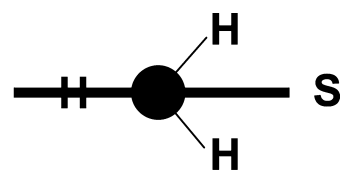

Staggered 
staggered configuration of the methyl group in $o$-toluidine can be attributed to both factors. There is a repulsive steric interaction of the in-plane hydrogen atom with the amino group, and the $\mathrm{C}_{2}-\mathrm{C}_{3}$ bond has a higher $\pi$-bond order than the $\mathrm{C}_{1}-\mathrm{C}_{2}$ bond. (The $\mathrm{C}_{1}-\mathrm{C}_{2}$ bond is $1.18 \mathrm{pm}$ longer than the $\mathrm{C}_{2}-\mathrm{C}_{3}$ bond.) The repulsive steric interaction is absent in $m$-toluidine, so the small preference for the eclipsed conformation of the methyl group in this molecule can be traced to the slightly higher $\pi$-bond order of the $\mathrm{C}_{2}-\mathrm{C}_{3}$ bond. (The $\mathrm{C}_{3}-\mathrm{C}_{4}$ bond is $0.02 \mathrm{pm}$ longer than the $\mathrm{C}_{2}-\mathrm{C}_{3}$ bond.)

Excitation of the two molecules to their $\mathrm{S}_{1}$ states has a significant impact on the methyl rotor potentials, owing to a quinoidal distortion of the aromatic ring. Thus, in $m$-toluidine, this results in a larger difference of the $S_{1} \pi$-bond orders of the adjacent $\mathrm{C}-\mathrm{C}$ bonds. The difference between the $\mathrm{C}_{2}-\mathrm{C}_{3}$ and $\mathrm{C}_{3}-\mathrm{C}_{4}$ bond lengths is now about $0.14 \mathrm{pm}$, an increase of 0.12 $\mathrm{pm}$. This further stabilizes the eclipsed form and produces a significant increase in the magnitude of the barrier height, $V_{3}\left(\mathrm{~S}_{0}\right)$ $=9.45 \rightarrow V_{3}\left(\mathrm{~S}_{1}\right)=322 \mathrm{~cm}^{-1}$. In $o$-toluidine, the difference between the $\mathrm{C}_{1}-\mathrm{C}_{2}$ and $\mathrm{C}_{2}-\mathrm{C}_{3}$ bond lengths is now $0.86 \mathrm{pm}$ on excitation of the $S_{1}$ state, a decrease of about $0.3 \mathrm{pm}$. Therefore, the staggered form is still favored in the $S_{1}$ state but there is a significant decrease in the magnitude of the barrier height, $V_{3}\left(\mathrm{~S}_{0}\right)=703 \rightarrow V_{3}\left(\mathrm{~S}_{1}\right)=44.9 \mathrm{~cm}^{-1}$. Some reduction in the magnitude of the repulsive term also would be expected, since all $\mathrm{C}-\mathrm{C}$ bonds in the $\mathrm{S}_{1}$ state are longer than in the $\mathrm{S}_{0}$ state. Thus, light-induced changes in the $\pi$-electron distributions of the aromatic rings have a major impact on the preferred geometries and dynamic behaviors of the attached methyl groups.

\section{Conclusions}

Rotationally resolved $\mathrm{S}_{1} \leftarrow \mathrm{S}_{0}$ fluorescence excitation studies of $o$ - and $m$-toluidine have been performed. Analysis of the results reveals that the amino group is the dominant substituent in the electronic transition; the $\mathrm{S}_{1} \leftarrow \mathrm{S}_{0}$ transition moment is essentially perpendicular to the position of attachment of this group in both molecules. The resulting $S_{1}$ states have significantly different $\pi$-electron distributions in the aromatic ring, compared to the ground states. As a consequence, the preferred configurations and potential barriers that govern the motion of the attached methyl groups in the two molecules are quite different.

Acknowledgment. We thank David F. Plusquellic (NIST) for the JB95 fitting program used for the data analysis and Jens-
Uwe Grabow (U. Hannover) for helpful discussions. This work has been supported by NSF (CHE-0615755).

\section{References and Notes}

(1) Spangler, L. H.; Pratt, D. W. In Jet Spectroscopy and Molecular Dynamics; Hollas, J. M., Phillips, D., Eds.; Chapman \& Hall: London, 1995; p 366-398 and references therein. 212.

(2) Okuyama, K.; Yoshihara, K.; Ito, M. Laser Chem. 1987, 7, 197-

(3) Tan, X. Q.; Majewski, W. A.; Plusquellic, D. F.; Pratt, D. W. J. Chem. Phys. 1991, 94, 7721-33.

(4) Tan, X. Q.; Clouthier, D. J.; Judge, R. H.; Plusquellic, D. F.; Tomer, J. L.; Pratt, D. W. J. Chem. Phys. 1991, 95, 7862-71.

(5) Tan, X. Q.; Pratt, D. W. J. Chem. Phys. 1994, 100, 7061-7.

(6) Borst, D. R.; Pratt, D. W. J. Chem. Phys. 2000, 113, 3658-3669.

(7) Alvarez-Valtierra, L.; Yi, J. T.; Pratt, D. W. J. Phys. Chem. 2006, $110 B, 19914-19922$.

(8) Alvarez-Valtierra, L.; Tan, X.-Q.; Pratt, D. W. J. Phys. Chem. 2007, $111 A, 12802-12809$.

(9) Majewski, W. A.; Pfanstiel, J. F.; Plusquellic, D. F.; Pratt, D. W. In Techniques of Chemistry; Rizzo, T. R., Myers, A. B., Eds.; Wiley \& Sons: New York, 1995; Vol. 23, pp 101-48.

(10) Plusquellic, D. F. Ph.D. Thesis, University of Pittsburgh, Pittsburgh, PA, 1992.

(11) Plusquellic, D. F.; Suenram, R. D.; Mate, B.; Jensen, J. O.; Samuels, A. C. J. Chem. Phys. 2001, 115, 3057-3067.

(12) Frisch, M. J.; Trucks, G. W.; Schlegel, H. B.; Scuseria, G. E.; Robb, M. A.; Cheeseman, J. R.; Montgomery, J. A. J.; Vreven, T.; Kudin, K. N.; Burant, J. C.; Millam, J. M.; Iyengar, S. S.; Tomasi, J.; Barone, V.; Mennucci, B.; Cossi, M.; Scalmani, G.; Rega, N.; Petersson, G. A.; Nakatsuji, H.; Hada, M.; Ehara, M.; Toyota, K.; Fukuda, R.; Hasegawa, J.; Ishida, M.; Nakajima, T.; Honda, Y.; Kitao, O.; Nakai, H.; Klene, M.; Li, X.; Knox, J. E.; Hratchian, H. P.; Cross, J. B.; Bakken, V.; Adamo, C.; Jaramillo, J.; Gomperts, R.; Stratmann, R. E.; Yazyev, O.; Austin, A. J.; Cammi, R.; Pomelli, C.; Ochterski, J. W.; Ayala, P. Y.; Morokuma, K.; Voth, G. A.; Salvador, P.; Dannenberg, J. J.; Zakrzewski, V. G.; Dapprich, S.; Daniels, A. D.; Strain, M. C.; Farkas, O.; Malick, D. K.; Rabuck, A. D.; Raghavachari, K.; Foresman, J. B.; Ortiz, J. V.; Cui, Q.; Baboul, A. G.; Clifford, S.; Cioslowski, J.; Stefanov, B. B.; Liu, G.; Liashenko, A.; Piskorz, P.; Komaromi, I.; Martin, R. L.; Fox, D. J.; Keith, T.; Al-Laham, M. A.; Peng, C. Y.; Nanayakkara, A.; Challacombe, M.; Gill, P. M. W.; Johnson, B.; Chen, W.; Wong, M. W.; Gonzalez, C.; Pople, J. A. Gaussian 03, rev. 6.0; Gaussian, Inc.: Wallingford CT, 2004

(13) Gordy, W.; Cook, R. L. Microwave Molecular Spectra, 3rd ed.; John Wiley \& Sons: New York, 1984.

(14) Tan, X. Q.; Majewski, W. A.; Plusquellic, D. F.; Pratt, D. W.; Meerts, W. L. J. Chem. Phys. 1989, 90, 2521-2.

(15) Herschbach, D. R. J. Chem. Phys. 1959, 31, 91-108.

(16) Kalkman, I. V. Ph.D. Thesis, Radboud University, Nijmegen, The Netherlands, 2008

(17) Watson, J. K. G. In Vibrational Spectra and Structure; Durig, J. R., Ed.; Elsevier: Amsterdam, The Netherlands, 1977; Vol. 6, pp 1-89.

(18) Plusquellic, D. F.; Pratt, D. W. J. Chem. Phys. 1992, 97, 89708976

(19) Sinclair, W. E.; Pratt, D. W. J. Chem. Phys. 1996, 105, 79427956

(20) Trinkaus, A. Diplom Thesis, University of Freiburg, 1967. 